\title{
A genetic register for Huntington's chorea in South Wales
}

\author{
PETER S HARPER, AUDREY TYLER, SUE SMITH, PAT JONES, \\ ROBERTG NEWCOMBE, AND VALERIE McBROOM
} From the Section of Medical Genetics, and the Department of Medical Statistics, Welsh National
School of Medicine, Cardiff

SUMMARY A regional genetic register for Huntington's chorea in South Wales is described, based on previous family studies in the area, which is one of high prevalence for the disorder. The primary role of the register is to help in the efficient delivery of services, including genetic counselling, to affected subjects and relatives, and to monitor changes in the population at risk. The mode of operation of the register is described and the essential importance of strict confidentiality is stressed.

Since 1973 a systematic study of Huntington's chorea (HC) has been in progress in South Wales. An initial survey attempting complete ascertainment was undertaken for the counties of Gwent and Glamorgan, comprising almost the whole of industrial South Wales with a population of 1.8 million. A high frequency of the disorder was found, with prevalence estimated at 7.6 per 100000 and heterozygote frequency close to 1 in $5000 .{ }^{1}$ Around 120 affected subjects with $\mathrm{HC}$ are alive in the study area at any one time, and the number of subjects in the area whose risk of developing HC exceeds 1 in 10 is around $1000 .^{2}$

Following the initial survey, a system of prospective surveillance was established for the family members at high risk, combining systematic genetic counselling with general support and with regular home visits to collect data concerning new births and other family changes. The preliminary results showing an apparent decline in high risk births have already been reported. ${ }^{3}$

Our prospective studies have only been made possible by the establishment of an efficient system of record keeping, which has in effect become a genetic register for $\mathrm{HC}$ in South Wales. We describe here the way in which the register evolved, its present mode of operation, its immediate and long term aims, and some of the special and general problems encountered in its management.

\section{The establishment and development of the register}

The initial survey of HC in South Wales recorded a

Received for publication 14 October 1981 considerable amount of clinical, genetic, and epidemiological information on a standardised protocol which was transferred to punched cards as the basis for subsequent analyses. Each kindred and each patient within a kindred was allocated a unique number, allowing kindred members to be linked in analyses. However, most of the items recorded were statistical, and in particular identifying items such as name and address were not computerised, so that this body of data, while extensive and largely complete, did not in itself form the basis for a register.

Concurrently with the initial survey, a card index file was developed for the practical purpose of recording basic information on family members and of locating their detailed records. A $12.5 \times 7.5 \mathrm{~cm}$ card size was used and information was restricted to the following items.

(1) Study number. The same unique seven digit number was used as for the survey analysis, the first three digits indicating kindred number, the fourth digit the generation number, and the last three digits the individual number within the particular generation.

(2) Full name.

(3) Date of birth.

(4) Address.

(5) Clinical status (affected or at risk; living or deceased).

(6) Prior genetic risk, for example $1 / 2,1 / 4$ (only for those at risk).

(7) Conditional genetic risk (that is, prior risk modified by age of the subject or of an unaffected parent at risk). 
(8) Name and address of family doctor.

Two identical sets of cards were made out, one being filed alphabetically, the other in order of kindred and individual number. The alphabetical file was further separated into those at risk and those affected.

Corresponding to the card index file were two other essential items in the record system.

(1) A file of pedigrees with numbers and names corresponding to the cards. This was particularly valuable in allowing an immediate visual connection to be made between a subject and other parts of the kindred, something that was much less obvious from the card index and detailed record files.

(2) Files containing correspondence, clinical notes, and other detailed material were arranged in order of kindred number and by family units within kindreds. Filing of such material strictly by individual number was not feasible, since individual letters were frequently concerned with multiple members of a family.

The above system, set up with no extra secretarial help and maintained solely by members of the Unit working with the families, provided a satisfactory basis for record keeping during the initial survey and the early stages of the subsequent prospective study (1974 to 1978). Frequent uses of the system included:

(1) Filing of new correspondence and clinical notes.

(2) Identification of new family members at risk.

(3) Telephone enquiries as to whether a particular patient or family member was already known to us.

(4) Retrieval of information for clinic and home visits.

(5) A rapid check on the risk status of family members.

From the above uses of the card index system it can be seen that although initially designed as a simple record system, it had in fact become a true register, aiming at completeness in recording information on all subjects known to be affected or at risk for HC in the study area, as well as aiming to keep this information updated.

By the end of 1978, however, signs were emerging that the system was no longer adequate. Particular problems included the following.

(1) Increased numbers of cards, with increased risk of misfiling. The peak number recorded by the file was 2310, of which 1440 were at risk.

(2) Confusion of people with the same surname. This is a particular problem in Wales, where the number of surnames is limited and where possession of the same surname only rarely implies a genetic relationship. Thus, the card index contained 92 people with the surname
Jones. Other common surnames included Davies (65), Williams (81), and Morgan (76). Since the first name as well as the surname was often identical, confusion was easy and was increased by the tendency of males to use an intermediate 'Bardic' name in preference to the first name.

(3) Updating of risk information. This proved difficult, since development of $\mathrm{HC}$ in one person would frequently alter the risks for a considerable number of other family members. Similarly, with the passage of time the gradual decline in risk for older members was tedious to record. Increasing numbers of instances were found where lack of updating seriously affected the accuracy of the risk estimate.

\section{Computerisation of the register}

In 1979 the decision was made to combine elements of the existing card index register and the body of information already computerised into a single register. The aims of the new register were principally:

(1) To allow the information on all members of the families to be recorded in a permanent, secure, yet accessible form.

(2) To provide a system where changes in family structure and genetic risks could be systematically and easily updated over a prolonged period.

(3) To assist the personnel involved in follow up of family members by providing rapidly available documentation on individuals, including lists of those needing visits, or where risks had become so low that visits were no longer required.

(4) To reduce to a minimum the clerical work involved in maintaining contact with the families.

(5) To provide statistical information on trends in prevalence, birth rate, migration, and other variables necessary to assess whether our long term preventive programme was having any significant effects on the population under surveillance.

It can be seen that the properties of this register were envisaged as providing a service to the families, rather than aiding research, though it was felt that the nature of the register would itself provide valuable data concerning $\mathrm{HC}$ and its possible prevention.

The data selected consisted of the identifying information on the index cards, together with the linking structure of the original computerised data, allowing new information on one individual to be related to that on others within the kindred. In particular, the system of risk estimation based on a life table method $^{4}$ was incorporated in such a way 
that development of the disease in one family member would automatically adjust the risk estimates for all other family members.

A conscious decision was made to exclude two items of information, name and address, from the main file. Instead this information was placed on a separate file, together with the unique numbers of individuals, which formed a link between the two files. The file containing name and address was subsequently destroyed, except for two copies within the Genetics Unit, and all further data entered on and produced by the main file contained no name and address. There were two reasons for this decision.

Firstly, the computer used for constructing and maintaining the register was not entirely dedicated to the Genetics Unit, though accessed from its own terminal within the unit. It was felt that as a general principle no identifying information should be allowed outside the Unit.

Secondly, the production of large numbers of listings and follow up forms inevitably increased the chance of loss of one of these. Without the identifying information on the form and without access to the linking file such information would be relatively meaningless if it fell into the wrong hands.

\section{Current operation of the register}

Since January 1980 information on all except a few newly diagnosed families has been placed on the register and the system of information updating and home based follow up has been entirely related to it. Data are entered and outputted through a terminal in the Genetics Department, which consists of a visual display unit with printing attachment. The discs containing the main file are maintained in the computing section of the Department of Medical Statistics, to which the terminal relates.

The main sequence of operation is shown in the figure. Essentially, the main body of data is held in the computer in a storage file and can be altered by new information, either entered directly on that individual or on another related individual.

Registration of new subjects, either born or identified after the setting up of the main file, is achieved by completion of a specific form containing the information otherwise provided by the original card system and survey data. A form is completed for each birth as soon as recognised, regardless of the fact that this individual will not require separate visits or be at risk of developing $\mathrm{HC}$ for a number of years.

When an updating visit is required, the fieldworker involved (an experienced nurse) requests a form, which is supplied by the computer with

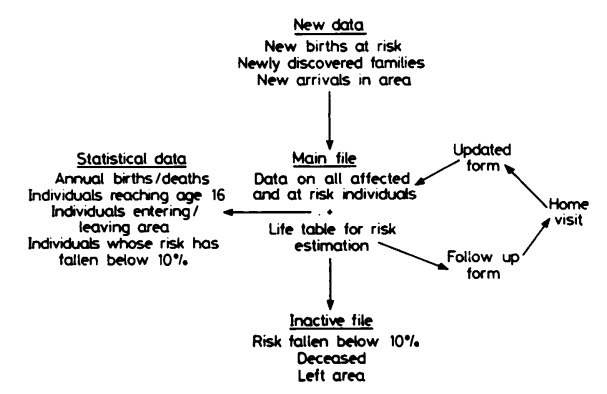

FIGURE Operation of the register.

current information entered on it. The fieldworker then supplies the name and address from the linking file, takes the form to the home visit, and records any changes on it, after which these are entered into the computer through the Genetics Department terminal to update the main file. The format is such that where no change has occurred only a tick is required, reducing the amount of writing necessary.

In addition to the individual follow up forms, a number of summary tables are produced at regular intervals, which are of considerable value. These include:

(1) An annual list of all subjects at risk reaching the age of 16. This allows consideration of genetic counselling for those previously considered to be too young to be given detailed information (though many will already have been partly or fully informed by their parents). The precise age at which full genetic counselling is given will, of course, depend on the wishes of the family, the maturity of the individual, and numerous other variables, but the production of an annual list ensures that those entering maturity are not overlooked.

(2) An annual list of those whose risk of developing HC has fallen below 1 in 10. This consists of elderly people with an affected parent and of younger people with an affected grandparent, but whose intervening parent is healthy. We have found it common for family members to be under the impression that their risk is much higher than it really is, and one potential danger of a register is that once on it, nobody can get off again! Although the risk figure of 1 in 10 is an arbitrary one, some 'cut off' point is required and an annual list of those whose risk has fallen below it is valuable. We make it a policy to pay a 'final' visit to such individuals to make sure they appreciate that the risk to themselves and their descendants is indeed small, while pointing out that the situation could alter (for example, by an 
elderly intervening parent developing the disorder), and also offering help should they wish to contact us in future.

(3) Individuals at risk leaving the area. This important group is likely to increase as the population becomes more mobile. Unemployment in South Wales may prove a major factor in such movement, just as the development of industry did in the origin of most of our present families. Some individuals move to other areas of Wales, where the genetics services are the same, but most migration is to England, where no systematic $\mathrm{HC}$ registers comparable with our own exist. Where possible, we attempt to keep in contact, or to inform colleagues in the appropriate regional centre (if the individual gives permission for this). There is little doubt, however, that unless a network of regional registers is to be developed, contact with a significant proportion of individuals will be lost.

\section{Staffing}

The current staffing of the register consists of one nurse fieldworker ( 30 hours per week), one part time medical officer ( 8 hours per week), and one clerical and records assistant ( 20 hours per week). Other staff, however, contribute considerably to the overall work on $\mathrm{HC}$, including the consultant in Medical Genetics and a research social worker, while consultation with other clinicians, notably in the Department of Neurology, is frequent. To set up a register in a Unit not already actively involved with $\mathrm{HC}$ families would entail increased staffing.

\section{Problems associated with the register}

These may be divided into specific operational difficulties and more general problems. The first category has been surprisingly lacking, apart from delay in the production and processing of forms in the early stages of the register. With the system now fully operational for more than a year, both the registration of new individuals and the follow up of family members has proceeded smoothly, and the staff involved have found the system easy to work with.

\section{Confidentiality}

Concern regarding the confidentiality of all recorded information has been a major concern from the outset of our studies on HC. The sensitive nature of much of this information and the severity of the disorder make it imperative that no identifying information concerning patients or relatives is disclosed to a third party, without express permission being given. We have frequently been approached with requests for information by various bodies, including social service departments, life insurance companies, driver licensing centres, and research workers in HC. We have made it an invariable rule not to divulge information without consulting the individual concerned, feeling that all information had been given voluntarily by patients and their families and their trust was the foundation of our work. Requests from other clinicians directly involved in medical care of the patients have been viewed in a different light and treated as would be the case for any other disease.

The policy regarding transfer of information has not in any way been altered by the establishment or computerisation of our register, but it has meant an increase in enquiries, balanced by an increase in referrals of possible new cases. In very few cases, in which the information requested has satisfied the above criteria, has it proved impossible to provide such information.

Regarding the security of the records themselves, it is likely that this has been increased by computerisation, though both card index and detailed records have always been kept in locked filing cabinets. The decision already mentioned not to allow identifying information on computerised data was made not so much because it was felt that security would be breached, but because it was felt that some individuals might feel uneasy about their identity being in a computer. Justifiable anxiety about computers and privacy in other fields of life might easily have had a 'backlash' effect and impaired the co-operation that we currently enjoy.

Access to the register data is confined to the Unit directly involved with the Huntington's chorea study, comprising one consultant and his associated medical staff, a nurse fieldworker and social worker, responsible for the family follow ups, and a clerk. There is no direct access by any other hospital clinical or records staff or by research workers.

Because of the lack of computerisation of identifying data and because our register is essentially an extension of our personal records system, we do not ask permission before placing an individual's name on the register, but do ask permission before releasing any information and to keep in regular contact. In this respect our register is fundamentally different from registers where information is entered and retrieved by more than one group of workers. ${ }^{5}$ The limitation of our register to a defined and compact geographical area means that the families are all known personally to us in a way that would not be possible were the register a national 
one, or maintained by people not intimately concerned with the clinical care of the families.

\section{Long term aims}

Establishment of a regional register for $\mathrm{HC}$ has not been an aim in itself, but has been a tool in our long term prospective study of the disorder in South Wales. There is no doubt that our aim of documenting trends in future prevalence in relation to genetic counselling and other preventive measures would be impossible without such a register, and it is likely that the effectiveness and completeness of the supporting services which we attempt to provide would be seriously impaired.

A further aim of establishing our register has been to demonstrate the feasibility of approaching the long term prevention of $\mathrm{HC}$ on a regional basis, and to encourage others to consider a comparable approach. While we would urge caution in view of the expense, time, and staff that are required to undertake such a project satisfactorily, we feel that regional centres such as our own, serving populations of 1 to 4 million people, are the natural level at which this type of service should function. To our knowledge, only one other region in the UK has embarked on a similar project.

\section{Conclusion}

The evolution of a regional register for Huntington's chorea in South Wales has taken place over a period of 8 years, during which time all known families in the area (122 kindreds at present) have been identified and studied intensively. The computerisation of the register has considerably aided our attempt to follow regularly and to provide genetic counselling for all subjects at high risk within the families, and we are currently maintaining contact with 986 such individuals.
During the time the register and its preceding record system has been in operation, a marked drop in high risk births, suggesting a decline in future incidence, has been recorded. While it is not possible to be certain that this drop is the direct result of the systematic genetic counselling and family support that we have given, it is certain that without the register we should not have been able to monitor the changes that have occurred.

We believe that if an attempt at the long term prevention of $\mathrm{HC}$ is to be made, a register such as our own is an essential part of such a programme. We have shown that it can function efficiently and be acceptable to families and we hope that as more effective diagnostic and therapeutic measures become available, the existence of the register will ensure that these are effectively applied.

\section{References}

1 Walker DA, Harper PS, Wells CEC, Tyler A, Davies K, Newcombe RG. Huntington's chorea in South Wales. A genetic and epidemiological study. Clin Genet 1981; 19:213-21.

2 Harper PS, Walker DA, Tyler A, Newcombe RG, Davies K. Huntington's chorea. The basis for long-term prevention. Lancet 1979 ;ii:346-9.

3 Harper PS, Tyler A, Smith S, Jones P. Decline in the predicted incidence of Huntington's chorea associated with systematic genetic counselling and family support. Lancet 1981 ;ii:411-3.

4 Newcombe RG. A life table for onset of Huntington's chorea. Ann Hum Genet 1981 ;45:375-85.

5 Emery AEH, Brough C, Crawford M, Harper PS, Harris R, Oakshott G. A report on genetic Registers. J Med Genet 1978;15:435-42.

Requests for reprints to Professor P S Harper, Section of Medical Genetics, Welsh National School of Medicine, Heath Park, Cardiff CF4 4XN. 